\title{
Circuit Equivalent to Rucklidge System
}

\author{
Jiri Petrzela \\ Department of Radio Electronics \\ Faculty of Electrical Engineering and Communications, Brno University of Technology \\ Brno, Czech Republic \\ petrzelj@feec.vutbr.cz
}

\begin{abstract}
This short report describes completely analog circuit realization of the so-called Rucklidge equations. Design process is systematic and supported by a circuitoriented numerical analysis (bifurcation diagrams and Lyapunov exponents) and real experimental verification via the oscilloscope screenshots. Oscillator is constructed such that the very simple one-to-one relations between mathematical model and circuit parameters is achieved. Proposed circuit solution uses off-the-shelf components only and provides independent continuous control of the internal parameters that can be varied within the large scale; a much wider than the real physical system that describes a double convection problem. Very good final agreement between theoretical assumptions (numerical integration) and laboratory measurement is achieved.
\end{abstract}

Keywords-analog computer; bifurcation diagram; chaotic oscillator; Lyapunov exponents; strange attractor

\section{INTRODUCTION}

Design of analog chaotic oscillators belongs to old topics. First dynamical system with associated chaotic behavior was constructed in the form of the lumped circuit in the middle of eighties and is named after its inventor as Chua's oscillator [1-3]. Since then, a huge number of both autonomous and driven chaotic system were constructed utilizing different synthesis methods. Probably the most straightforward approach how to realize set of ordinary differential equations is concept known as analog computer. Only three building blocks are required for the circuit design: inverting integrator, differential amplifier and two-port having a prescribed nonlinear input-output characteristic [4, 5]. Complex nonlinearity can be implemented using A/D and D/A converters using digital sub-circuit [6]. Chaotic system is, by following rules of the analog computers, usually implemented in voltage mode, i.e. active elements are standard operational amplifiers. Thus, final network is mostly quite complicated but using only commercially available and cheap active elements. Another approach commonly utilized for circuit synthesis of a dynamical system is directly derived from application of the first Kirchhoff's law [7]. Individual differential equations describe sum of all currents flowing through grounded capacitors. It means that state variables are voltages across these capacitors. Recently, FPAA realizations of the chaotic systems become more common [8-10]. Development kits are quite cheap, system interface is user friendly and a complete circuitry implementation can be done within a few hours. Moreover, parameters of original math model can be adjusted arbitrarily.

Research described in this paper was financially supported by Grant Agency of Czech Republic under project no GA19-22248S.
Origin of the Rucklidge system itself is dated back to the early nineties [11]. This third-order autonomous deterministic dynamical system describes a nonlinear double convection problem in fluid mechanics. Global behavior of this circuit was studied in several papers, turns to be rich including chaotic transients as well as a robust chaotic motion. Aim of this brief paper is to provide the interested readers with the circuit tool to further continue investigations of this very simple but unique set of the ordinary differential equations.

\section{MATHEMATICAL MODEL AND BACKGROUND}

Famous Rucklidge dynamical system is described by following set of the ordinary differential equations

$$
\begin{aligned}
& \frac{d}{d t} x=a \cdot x+b \cdot y+y \cdot z \\
& \frac{d}{d t} y=x \quad \frac{d}{d t} z=-z+y^{2}
\end{aligned}
$$

where $a, b$ are internal parameters and state variables will be easily measurable node voltages. The reference chaotic trajectories can be obtained by using Mathcad and build-in fourth order Runge-Kutta method with a fixed time step $10^{-2}$ and final time $10^{3}$. Corresponding results are provided by means of Fig. 1 .
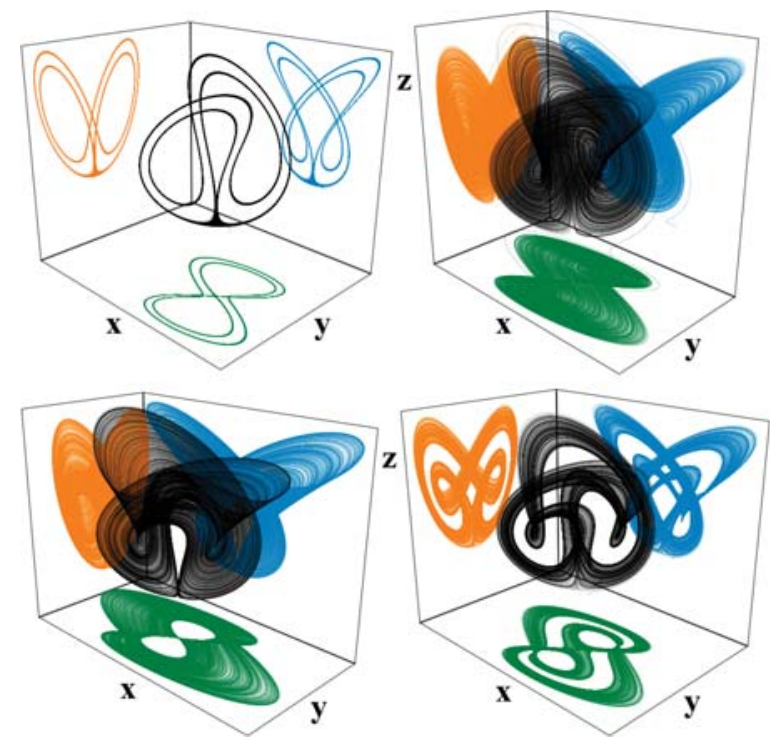

Figure 1. Three-dimensional perspective view on the numerically integrated typical strange attractor generated by Ruckligde system: $a=2, b=4$ (upper left picture), $a=2, b=6$ (upper right picture), $a=1.3, b=6$ (lower left image) and $a=1.3, b=4$ (lower right plot). 
From the viewpoint of upcoming practical realization, it is important to check basins of attraction leading to some bounded state space attractor; especially chaotic one. During these calculations in a rough grid defined around origin it turns out that region of attractivity fills entire state space with exception of origin itself. That seems to be advantageous because we always end-up in the chaotic regime of a circuit during measurement. Another analysis that needs to be done is specification how a dynamical motion changes with the large-scale variations of internal system parameters. The largest Lyapunov exponent (LLE) belongs to the most wellestablished quantifiers of dynamical flows. Its positive value indicates that behavior is sensitive to changes of the initial conditions, i.e. it is truly chaotic. Calculation of LLE can be delayed on the orbit such that we are able to completely omit chaotic transients. Using this plot, we can determine if areas with positive LLE are wide enough such that strange attractor will be robust and experimentally observable. On the other hand, the narrow regions suggest possible problems with sudden strange attractor disappearing due to the uncontrolled parameter variations. For the outputs mentioned above please consult Fig. 2. Finally, pair of one-dimensional bifurcation diagrams has been calculated in order to support experimental observation of the route-to-chaos scenarios via a continuous hand-made change of both potentiometers; see Fig. 3. These can be considered as high-resolution plots because of very small parameter step $10^{-3}$. Individual cross-sections that form these plots are Poincaré sections taken as planes $x=0$ while $y$ state variable is plotted. Note that regions of a chaotic behavior alternate with the several periodic windows.
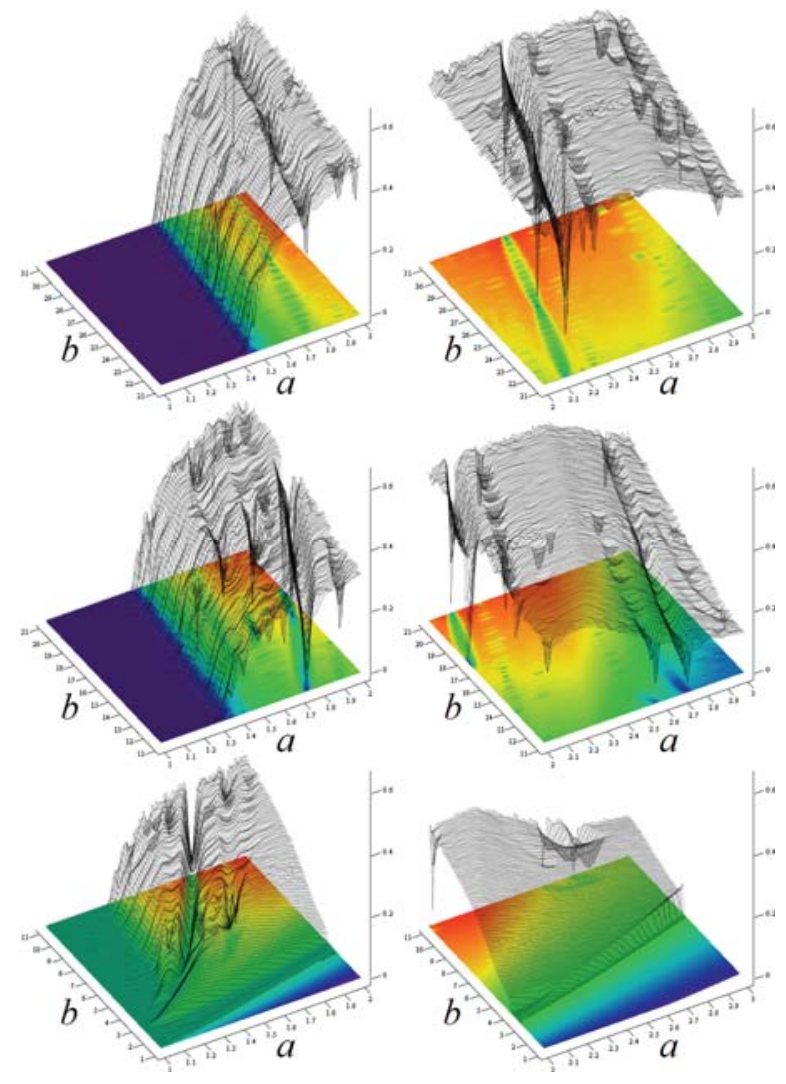

Figure 2. Rainbow-scaled surface-contour plot of LLE as function of both system parameters with small step 0.01 , color scale: -0.36 (dark blue), 0 (green), 0.32 (yellow) and 0.65 (red). Each contour plot has its own auto-scale in vertical direction, Mathcad program.
Deformation of vector field under dynamical flow is visible in Fig. 4. In this numerical output sensitivity of Rucklidge system to choices of the initial conditions was tested.

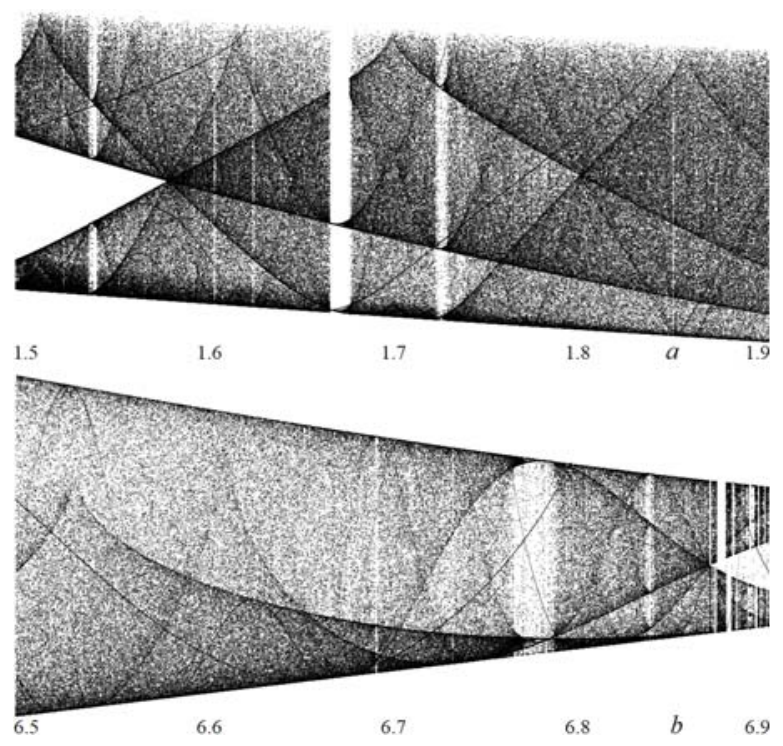

Figure 3. 1D bifurcation diagram calculated with respect to parameter $a$ (upper plot) and $b$ (lower plot), program Mathcad

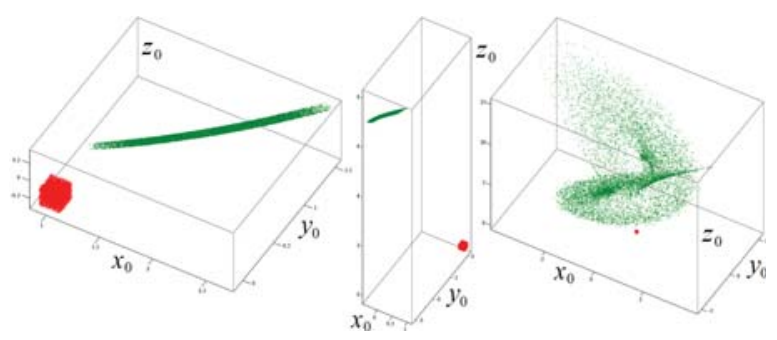

Figure 4. Sensitivity of Rucklidge dynamical system to changes of $10^{4}$ initial conditions located in a cube centered in the position $(1,0,0)^{\mathrm{T}}$ with edge 0.1 (red dots): final states (green dots) after short-time evolution equals to 1 (left plot), 10 (middle graph) and long-time evolution 100 (right image), program Mathcad.

\section{CIRCUIT REALIZATION}

By following the concept of the analog computers, dynamical system (1) is realized by oscillator given in Fig. 5. Describing set of the differential equations can be expressed as

$$
\begin{aligned}
& C_{1} \frac{d}{d t} v_{X}=-\frac{v_{X}}{R_{1}}+\frac{v_{Y}}{R_{2}}+\frac{K_{2}}{R_{6}} v_{Y} v_{Z} \\
& C_{2} \frac{d}{d t} v_{Y}=\frac{v_{X}}{R_{3}} \quad C_{3} \frac{d}{d t} v_{Z}=-\frac{v_{Z}}{R_{4}}+\frac{K_{1}}{R_{5}} v_{Y}^{2}
\end{aligned}
$$

Fundamental time constant of this oscillator is chosen to be $\tau=10^{4} 10^{-8}=100 \mu \mathrm{s}$ and $K_{i}=0.4$ represents internal scaling constant associated with $i$-th analog multiplier AD633. Transfer function of this four-quadrant active element is $V_{W}=K\left(V_{X 1}-V_{X 2}\right)\left(V_{Y 1}-V_{Y 2}\right)+V_{Z}$, i.e. versatile for many practical applications. Very high impedances of input terminals do not introduce error terms into describing differential equations (no leakage current). Individual inverting summing integrators are realized by cheap integrated circuit TL084. 
Chaotic behavior can be localized within dynamics of designed oscillator a following list of the numerical values of the passive circuit components: $C=2.2 \mathrm{nF}$, $R_{1}=5 \mathrm{k} \Omega, R_{2}=2 \mathrm{k} \Omega, R_{3}=R_{4}=10 \mathrm{k} \Omega$ and $R_{5}=R_{6}=1 \mathrm{k} \Omega$. Time constant of this oscillator can be changed simply by multiplication of each capacitor by the real number lower than one.

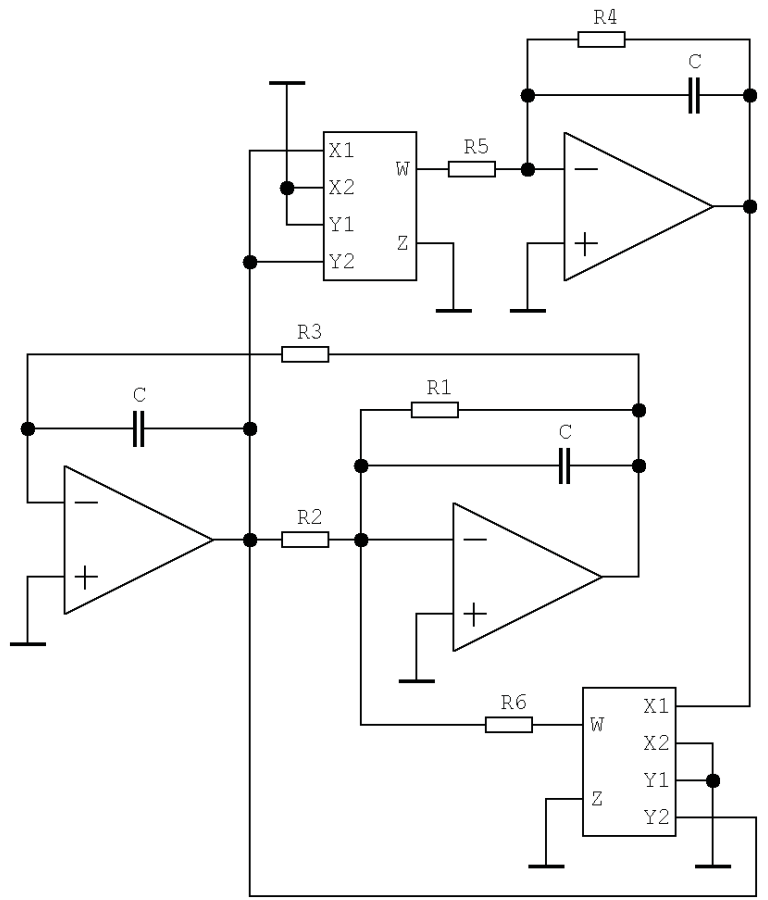

Figure 5. Circuitry implementation of Rucklidge dynamica system strictly following a concept of the analog computers.

\section{Simulation AND MEASUREMENT}

Figure 6 shows a computer-aided simulation result for mentioned circuit configuration.

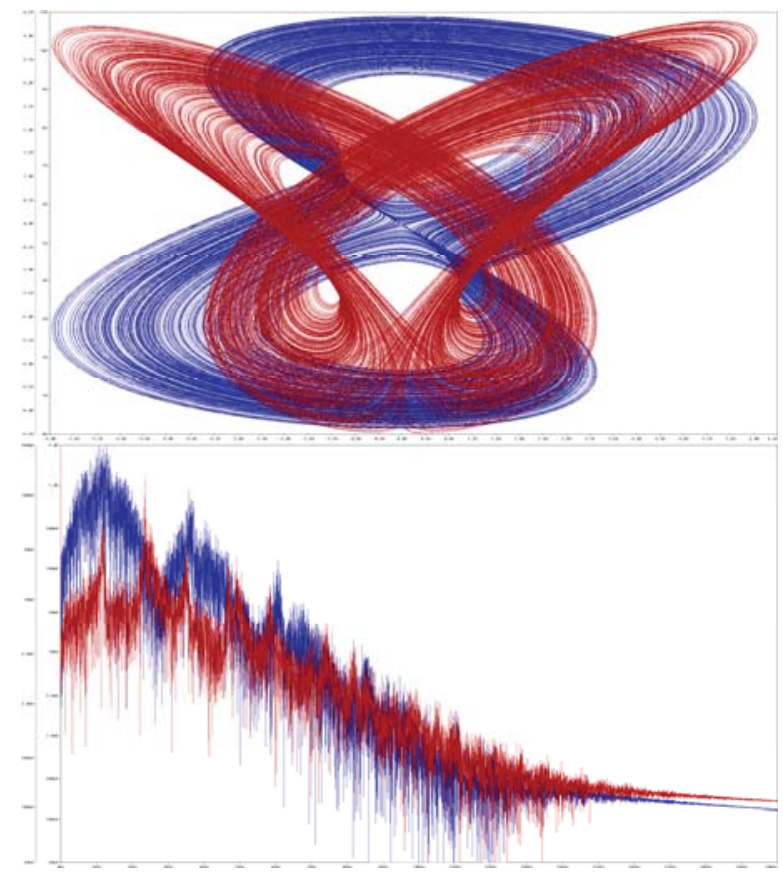

Figure 6. Orcad Pspice based simulation results: $v_{X}$ vs $v_{Y}$ (blue) and $v_{X}$ vs $v_{Z}$ (red) plane projection of the typical chaotic attractor (upper plots), generated chaotic signal $v_{X}$ (blue) and $v_{Y}$ (red) visualized in the frequency domain (lower plots).
Simulation profile was set to final time $100 \mathrm{~ms}$ and maximum allowed time step $1 \mu$ s. Note that strange attractor plotted in $v_{X}-v_{Y}$ Monge projection strongly resembles the famous double-hook attractor $[12,13]$. However, this circuit has only one fixed point located at origin; other two exist only for negative value of $b$.

Double-hook attractor was originally generated by piecewise-linear Chua's oscillator with inner segment having local geometry formed by three eigenvectors; i.e. equilibrium point located at origin has three real eigenvalues (two negative and one positive). However, analyzed Rucklidge mathematical model is completely different. Sophisticated math analysis, both numerical and analytical, such as that presented in [14], goes far behind allowed length of this paper. Here we can find place for future research.

Note that frequency spectrum has a Gaussian-like distribution and significant frequency components of the generated chaotic signals fall within audio range. Thus, route-to-chaos scenario can be heard using some acoustic transducer.

True experimental verification of a proposed novel chaotic oscillator is demonstrated by means of Fig. 7, Fig. 8, Fig. 9 and Fig. 10. First three figures show the limit cycles and subsequent chaotic behavior generated by Rucklidge system in fundamental plane projections. Figure 10 provides several oscilloscope screenshots of generated chaotic signals in the time domain with the different time scales.

Designed chaotic oscillator can be used to model double convection fluid dynamics especially if largescale fluctuations of the fundamental parameters need to be considered and/or analyzed. These quantities are represented and can be varied via a change of resistors (potentiometers) $R_{1}$ and $R_{2}$.

Author of this article believes that designed chaotic oscillator given in Fig. 5 belongs to the simplest and very robust direct electronic equivalent to the ordinary differential equations (1). However, circuit synthesis is task with many correct solutions. Mathematical model (1) can be realized as a current-mode circuit (i.e. state variables are currents) by adopting approach described in [15]. By processing currents instead of voltages, we can move fundamental frequency generated by system toward the upper frequency bounds; probably up to the hundreds of $\mathrm{kHz}$.
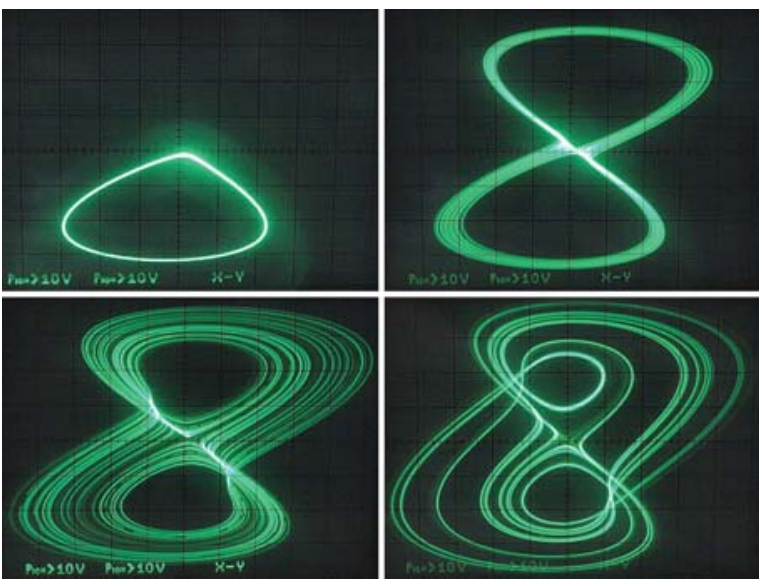

Figure 7. Measured state attractors plotted in $v_{X}$ vs $v_{Y}$ projection. 

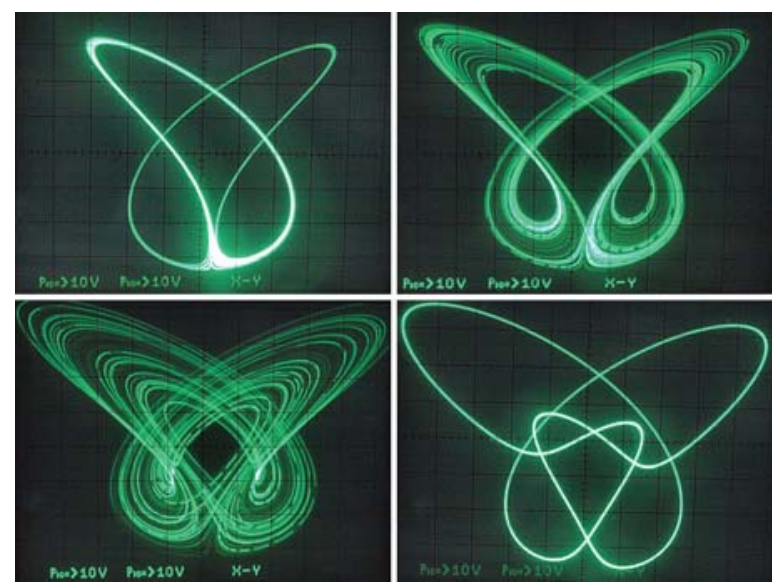

Figure 8. Measured state attractors plotted in $v_{X}$ vs $v_{Z}$ projection.
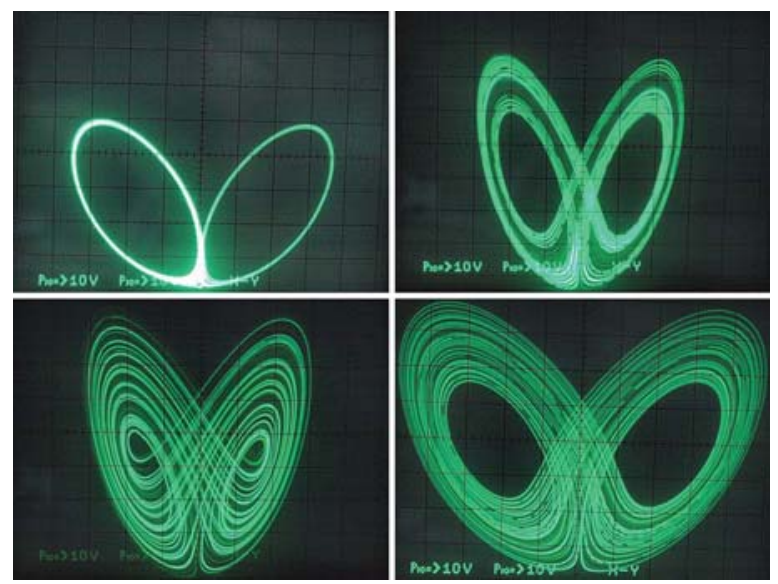

Figure 9. Measured state attractors plotted in $v_{Y}$ vs $v_{Z}$ projection.
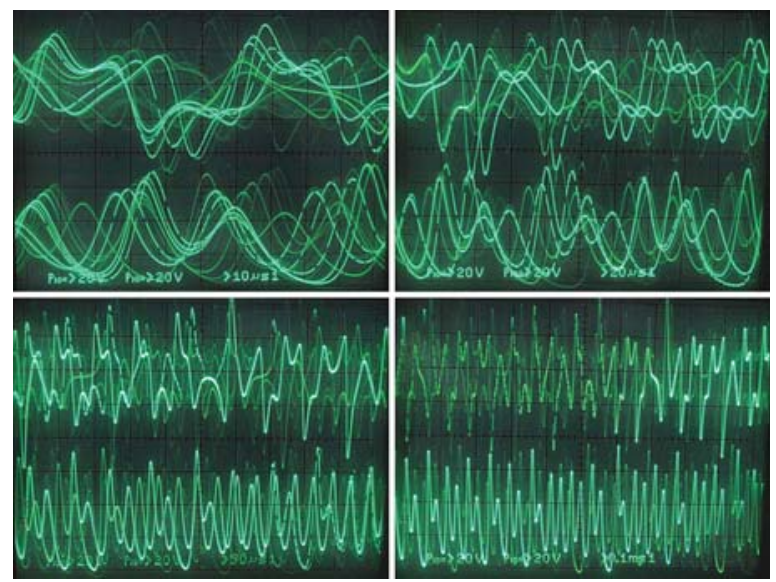

Figure 10. Chaotic waveforms in time domain with different scales.

\section{DISCUSION AND CONCLUSION}

This paper presents a simple fully analog circuitry realization of the Rucklidge system known from fluid dynamics. Experiments prove that designed electronic circuit represents precise model and can be used for further investigations of double convection dynamics.
A more comprehensive literature survey can also reveal possibilities how to implement designed chaotic oscillator in the practical applications such as secure communication systems [16], chaotic masking [17] or modulation [18].

\section{REFERENCES}

[1] L. O. Chua, M. Komuro, T. Matsumoto, "The double scroll family," IEEE Trans. on CAS I, vol. 33, no. 11, pp. 1073$1117,1986$.

[2] L. O. Chua, G. N. Lin, "Canonical realization of Chua's circuit family," IEEE Trans. on CAS I, vol. 37, no. 7, pp. 885902, 1990.

[3] Ch. Silva, L. O. Chua, "The overdamped double-scroll family," International Journal of Circuit Theory and Applications, vol. 16, no. 3, pp. 233-302, 1988.

[4] J. Petrzela, Z. Hrubos, T. Gotthans, "Modeling deterministic chaos using electronic circuits," Radioengineering, vol. 20, no. 2, pp. 438-444, 2011.

[5] M. Itoh, "Synthesis of electronic circuits for simulating nonlinear dynamics," International Journal of Bifurcation and Chaos, vol. 11, no. 3, 2001, pp. 605-653.

[6] T. Gotthans, J. Petrzela, "Experimental study of the sampled labyrinth chaos," Radioengineering, vol. 20, no. 4, 2011, pp. 873-879.

[7] J. Petrzela, "Electronically tunable analog chaos converters," In Proceedings of $35^{\text {th }}$ International Conference on Telecommunications and Signal Processing, Prague (Czech Republic), pp. 390-396, 2012.

[8] F. A. Dalkiran, J. C. Sprott, "Simple chaotic hyperjerk system," International Journal of Bifurcation and Chaos, vol. 26, no. 11, pp. 1650189, 2016.

[9] R. Kilic, F. Y. Dalkiran, "FPAA-based programmable implementation of a chaotic system characterized with different nonlinear functions," In Proceedings of International Symposium on Nonlinear Theory and Its Applications, Budapest (Hungary), pp. 132-135, 2008.

[10] R. Caponetto, A. Di Mauro, I. Fortuna, M. Frasca, "Field programmable analog arrays to implement programmable Chua's circuit," International Journal of Bifurcation and Chaos, vol. 15, no. 5, pp. 1829-1836, 2005.

[11] A. M. Rucklidge, "Chaos in models of double convection," Journal of Fluid Mechanics, vol. 237, pp. 209-229, 1992.

[12] C. P. Silva, "The overdamped double-scroll family. Part I: piecewise-linear geometry and normal form," International Journal of Circuit Theory and Applications, vol. 16, no. 3, pp. 233-302, 1988.

[13] P. Bartissol, L. O. Chua, "The double hook" IEEE Transactions on Circuits and Systems, vol. 35, no. 12, pp. 1512-1522, 1988.

[14] M. Guzan, "Variations of boundary surface in Chua's circuit," Radioengineering, vol. 24, no. 3, pp. 814-823, 2015.

[15] J. Petrzela, T. Gotthans, M. Guzan, "Current-mode network structures dedicated for simulation of dynamical systems with plane continuum of equilibrium," Journal of Circuits, Systems and Computers, vol. 27, no. 9, 2018, pp. 1-39.

[16] M. Itoh, "Spread spectrum communication via chaos," International Journal of Bifurcation and Chaos, vol. 9, no. 1, 1999, pp. 155-213.

[17] K. M. Cuomo, A.V. Oppenheim, "Circuit implementation of synchronized chaos with applications to communications," Physical Review Letters, vol. 71, no. 1, 1993, pp. 65-68.

[18] K. M. Cuomo, A.V. Oppenheim, "Synchronization of lorenzbased chaotic circuits with applications to communications," IEEE Transactions on CAS-II: Analog and Digital Signal Processing, vol. 40 , no. 10, 1993, pp. 626-633. 